\title{
Successful treatment of a childhood synovitis, acne, pustulosis, hyperostosis and osteitis (SAPHO) syndrome with subcutaneous methotrexate: A case report
}

\author{
Meltem Akçaboy, Sevcan Azime Bakkaloğlu-Ezgü, Bahar Büyükkaragöz, Emel Isıyel, \\ Yasar Kandur, Enver Hasanoğlu, Necla Buyan \\ Division of Pediatric Nephrology, Department of Pediatrics, Gazi University Faculty of Medicine, Ankara, Turkey. \\ E-mail: meltemileri@yahoo.com \\ Received: 14th October 2016, Accepted: 23rd December 2016
}

\begin{abstract}
SUMMARY: Akçaboy M, Bakkaloğlu-Ezgü SA, Büyükkaragöz B, Isıyel E, Kandur Y, Hasanoğlu E, Buyan N. Successful treatment of a childhood synovitis, acne, pustulosis, hyperostosis and osteitis (SAPHO) syndrome with subcutaneous methotrexate: A case report. Turk J Pediatr 2017; 59: 184-188.

SAPHO syndrome (synovitis, acne, pustulosis, hyperostosis and osteitis) is defined as a syndrome that is related to various osteoarticular manifestations and chronic dermatological conditions especially severe acne. SAPHO syndrome is a rare and unusual clinical entity in childhood and treatment choices are variable. We report an 11-year-old girl who suffered from SAPHO syndrome and successfully treated with subcutaneous methotrexate. We report our case in order to take attention to this rare clinical condition in evaluating patients and also to point out that treatment options beyond biologic agents should be the first line treatment in childhood.
\end{abstract}

Key words: children, methotrexate, SAPHO syndrome, treatment.

The acronym SAPHO (synovitis, acne, pustulosis, hyperostosis and osteitis) represents a syndrome characterized by the variable association of osteoarticular manifestations and various chronic dermatological conditions, particularly palmoplantar pustulosis and severe acne ${ }^{1}$. Although there have been case series of adults who have been followed up by the diagnosis of SAPHO syndrome ${ }^{2,3}$, there are few cases in childhood as case presentations ${ }^{4,5}$. The diagnosis of the SAPHO syndrome is based on exclusion of infectious arthritis, osteomyelitis, and tumoral conditions of the bone; and the presence of at least one of four diagnostic criteria proposed by Benhamou et $\mathrm{al}^{6}$.

Here, we present an adolescent girl who was diagnosed as SAPHO syndrome and has been followed up for two years in remission with subcutaneous methotrexate therapy.

\section{Case Report}

An 11-year-old girl admitted to our hospital with a history of pain on her left thigh, both ankles, left hip, spine and on sternum especially when taking breaths for 20-days and swelling on both ankles for two days. She was not even able to move or walk due to severe pain. She had lost $4 \mathrm{~kg}$ during the last 3 weeks. She did not have fever or any trauma. Family history and past medical history was unremarkable. Physical examination revealed pustuloform acne on the face, and pustular lesions on the whole body (Fig. 1), Sternal tenderness, ankle swelling and reduced motility of the vertebral column were remarkable. In her laboratory evaluation, erythrocyte sedimentation rate was $105 \mathrm{~mm} /$ hour and high sensitive CRP was $6.23 \mathrm{mg} / \mathrm{dl}(0-$ $0.2 \mathrm{mg} / \mathrm{gl}$ ). All other rheumatologic parameters including rheumatoid factor and autoantibodies were within normal ranges or negative. She was negative for HLA-B27. Full septic screens were negative. Cultures of the pustular lesions were negative. Conventional X-rays showed periostal reactions on the head of femur and osteitis on the bilateral sacroiliac bones. MRI showed pathologic signals and contrast uptake on L1 vertebra, sacrum, left sacroiliac bones and the adjacent soft tissues suggesting malignancy (Fig. 2, 3). Nuclear bone scans demonstrated 
multiple enhanced signal intensities on left sacroiliac region, left acetabular region and head of femur that were consistent with arthro-osteitis (Fig. 4). She was searched for autoimmune thyroiditis and inflammatory bowel disease and screenings were negative. Bone biopsy was done for differential diagnosis, especially to rule out malignancy, and it showed nonspecific subacute osteomyelitis by the infiltration of polymorphonuclear cells without any evidence of infection. After excluding infectious or malignant diseases, the patient was diagnosed as SAPHO syndrome and combined therapy [non-steroidal anti-inflammatory drugs (NSAID)-(naproxen sodium) (25 mg/kg/day), sulfasalazine $(50 \mathrm{mg} / \mathrm{kg} /$ day $)$ and prednisolone
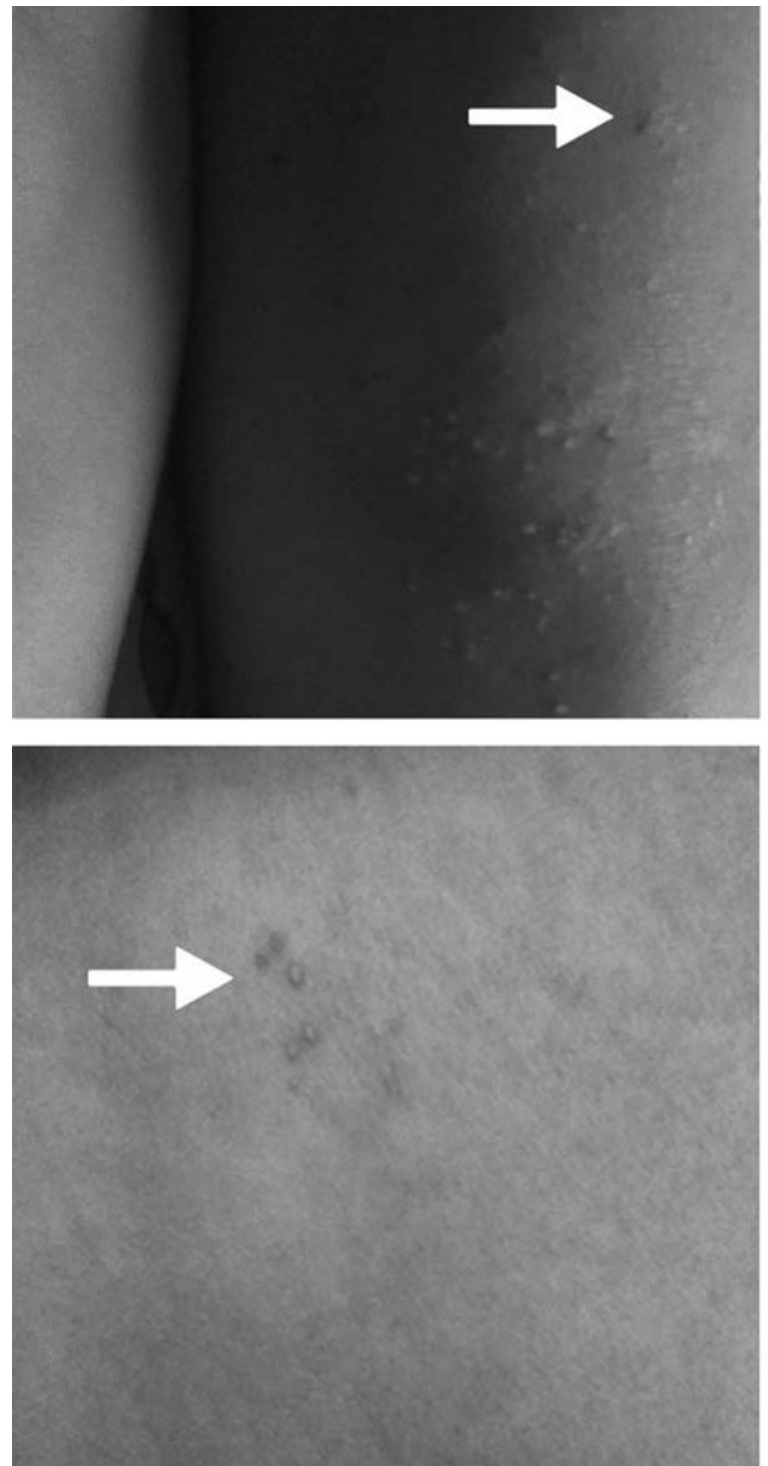

Fig. 1. Pustular lesions on different parts of the body.
(0.5 mg/kg/day)] was started. She responded dramatically to the treatment in the second week. ESR and CRP returned to normal ranges in a month gradually. The patient was discharged and followed on a regular basis in outpatient clinic. Corticosteriod was tapered and stopped by the third month. She did well by NSAID and sulfasalazine treatment only for a month and readmitted for generalized pain on the whole body. ESR and CRP were found to be high by $100 \mathrm{~mm} /$ hour and $42.7 \mathrm{mg} /$ dl (normal ranges: 2-6 mg/dl), respectively. Due to exacerbated symptoms, methotrexate $10 \mathrm{mg} / \mathrm{m}^{2} /$ week was added to the treatment. Because of the gastrointestinal intolerance to oral methotrexate, treatment was switched
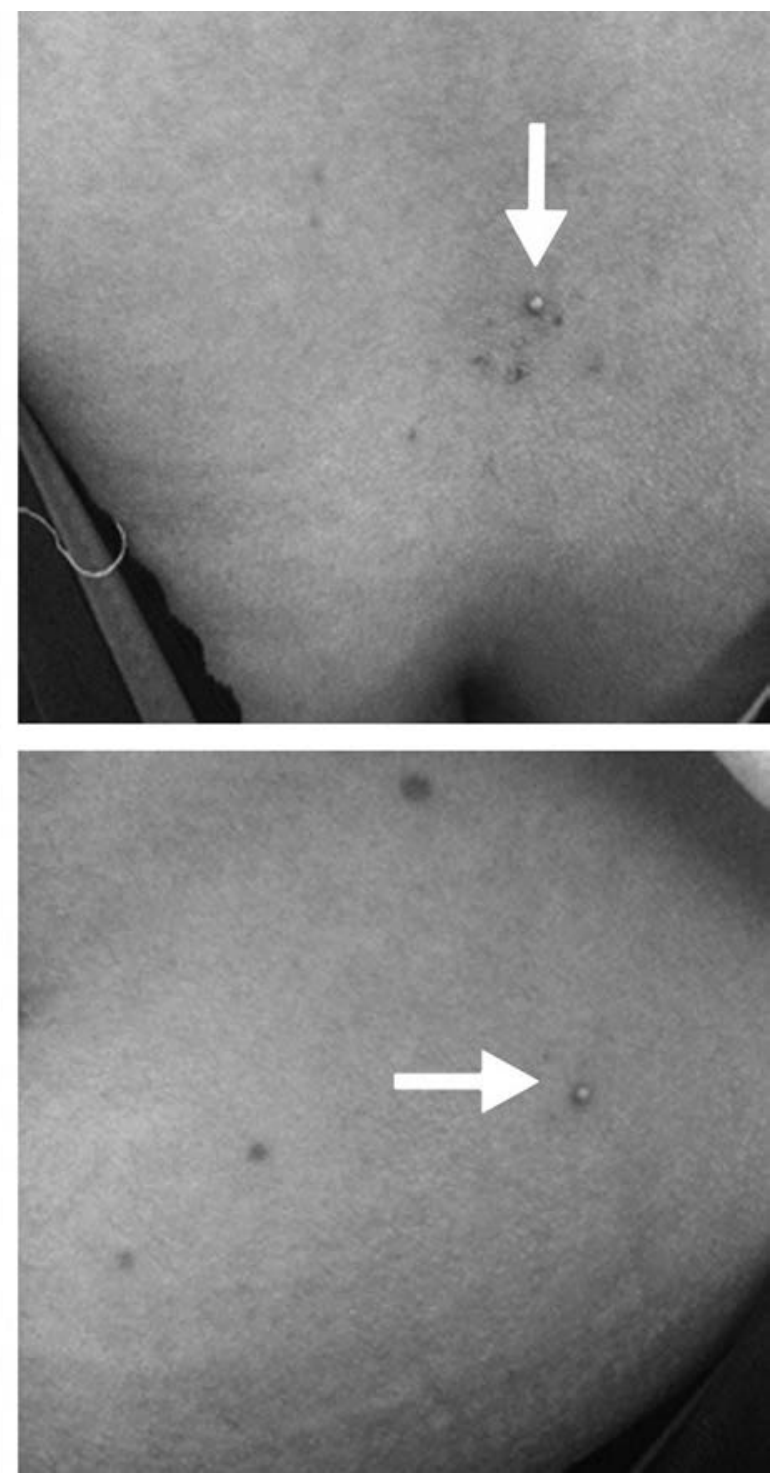

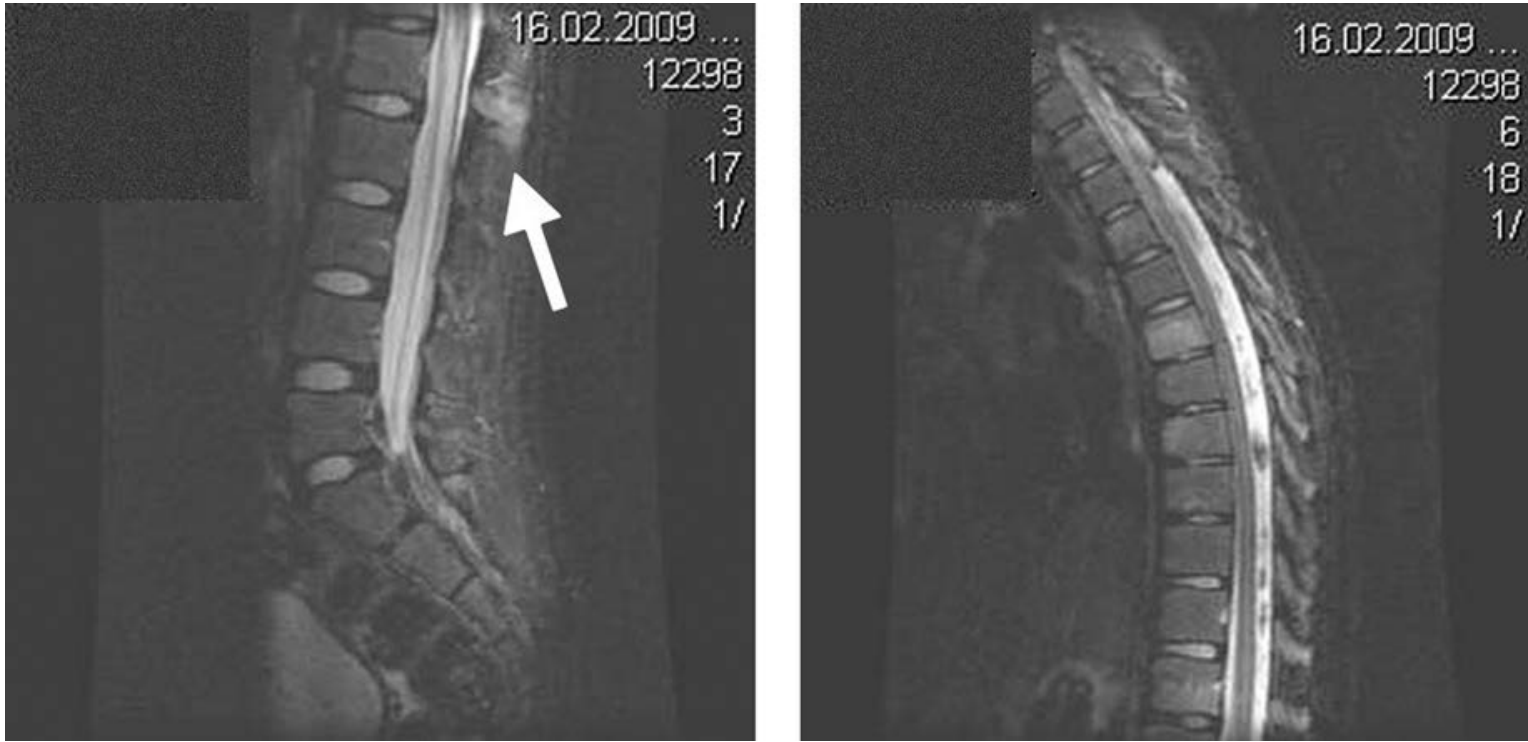

Fig. 2. Coronal T2-weighted magnetic resonance imaging showing pathological signal on the spinal process of L1 (arrow).
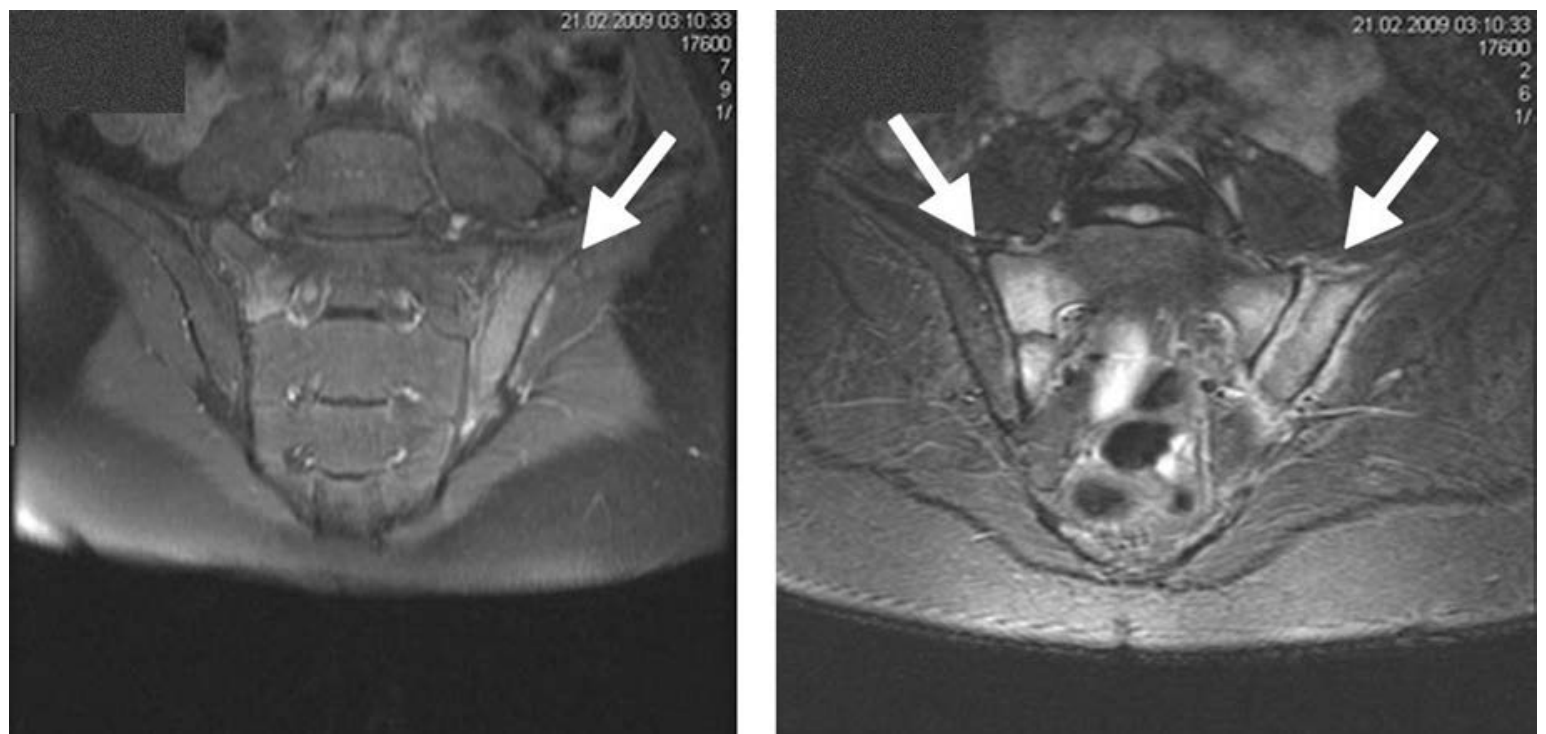

Fig. 3. Magnetic resonance imaging showing pathological signals and contrast uptake on sacrum, left sacroiliac bones and the adjacent soft tissue (arrows).

to subcutaneous methotrexate with the same dose after a month. In addition to clinical improvement, ESR and CRP turned to normal by the second month. After 6 months under this therapy, nuclear bone scan also improved remarkably (Fig. 4). She is being followed up without any symptoms under the treatment for two years without any symptoms or activity.

Written informed consent for the publication of this case report and any accompanying images were obtained from the patient's parents.

\section{Discussion}

SAPHO syndrome, characterized by a variable combination of osteoarticular and cutaneous manifestations, is highly rare in children ${ }^{4,5,7}$. The following features are reported to be characteristic for SAPHO syndrome: (a) local bone pain with gradual onset, (b) multifocal lesions, especially in tubular long bones and spine, (c) failure to cultivate an infectious organism, (d) a protracted course for years with exacerbations and improvement with antiinflammatory drugs and (e) skin conditions 

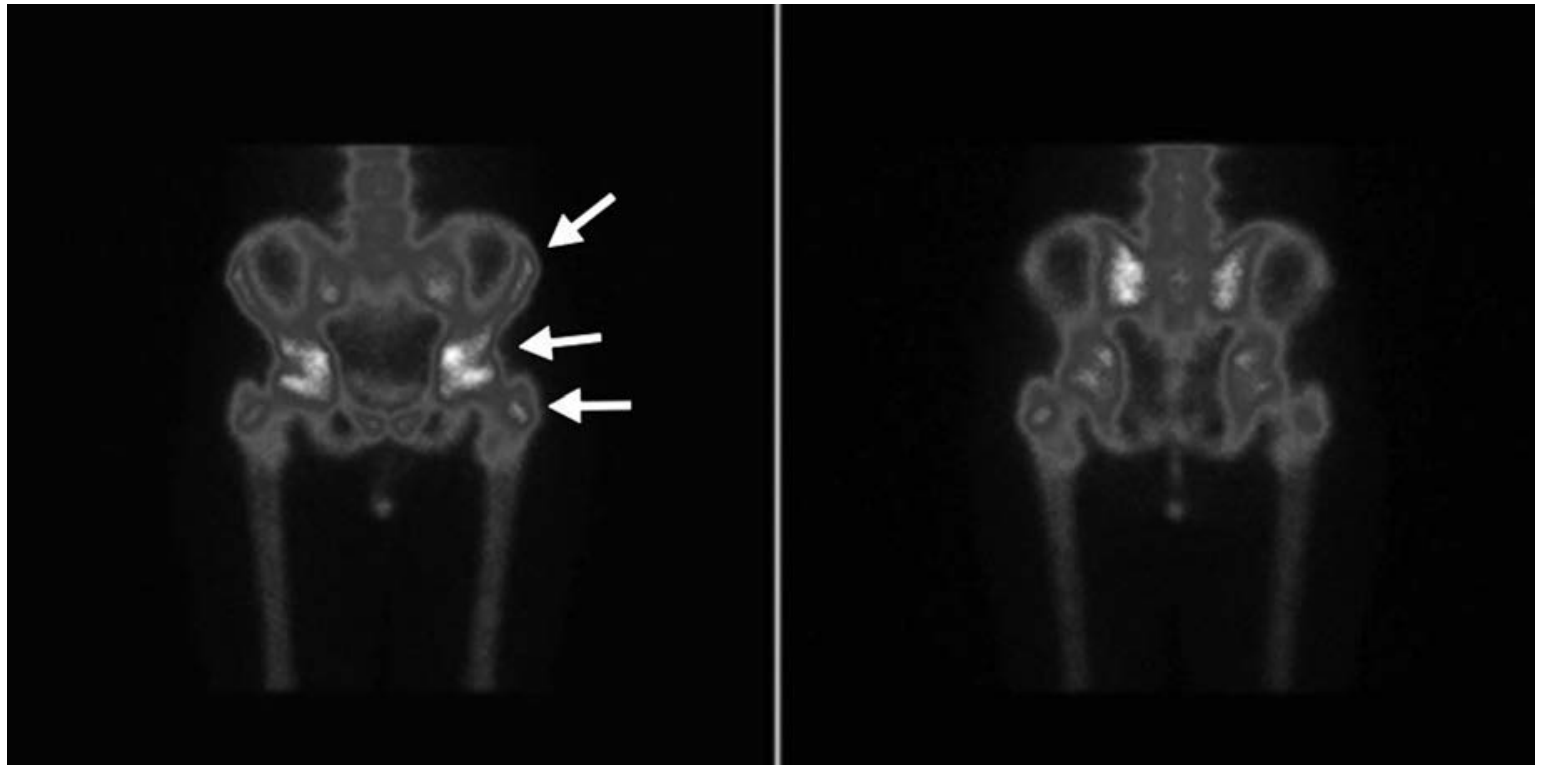

Fig. 4. Bone scintigraphy on admission demonstrating multiple enhanced signal intensities on the left sacroiliac region, left acetabular region and head of femur (on the left), and 6 months after the treatment (on the right).

sharing the common denominator of being filled with neutrophils, mostly palmoplantar pustulosis, non palmoplantarpustulosis, psoriasis vulgaris or severe acne ${ }^{8}$. Our case met all criteria for the diagnosis.

Osteitis on chest wall, spine, pelvic girdle and peripheral bones and hyperostosis are striking features that can be observed in any skeletal segment ${ }^{2,7}$. CT and MRI are helpful in locating the lesions and providing information on adjacent soft tissues, but cannot distinguish between SAPHO lesions, osteomyelitis and malignancy ${ }^{9}$. Bone scintigraphy is important in diagnosing SAPHO syndrome, particularly for detecting early bone involvement ${ }^{10}$. Bacterial cultures are usually negative ${ }^{2}$ but both Staphylococcus aureus and Propionibacterium acnes (P. acnes) have been isolated ${ }^{11}$ in some cases, but the correlation between the pathogenesis and these agents are still controversial. Bone biopsy is often used in an attempt to establish a diagnosis, especially in cases with extraaxial bone lesions ${ }^{12}$. Bone histopathology is variable, with early lesions characterized by the presence of polymorphonuclear infiltrate. Laboratory findings are usually of little value, and include mild, non-specific elevation of various inflammatory indices ${ }^{12}$. We evaluated our patient by MRI, and bone scintigraphy, but we needed a bone biopsy to rule out malignancy. The presence of polymorphonuclear infiltrate in a culture negative bone biopsy material mainly suggested SAPHO syndrome.

Nosologic framing of SAPHO syndrome is still a matter of debate ${ }^{2,6}$. Different stimuli have been implicated as inciting factors. Infectious, hypersensitivity and autoimmune processes have all been proposed, but are unconfirmed ${ }^{2}$. There is growing evidence that an exaggerated response to intestinal bacteria mediated by the NOD2/CARD 15 (nucleotide-binding oligomerization domain protein 2 /caspase recruitment domain 15) system in the inflammasome leading to a nuclear factor-kappa-B overactivation may be involved in SAPHO syndrome ${ }^{13}$, which proposes a polygenic auto-inflammatory disease pathogenesis ${ }^{14}$. Recently in a case of SAPHO syndrome, dysregulation of extracellular ATPdependent $\mathrm{P}_{2} \mathrm{X}_{7}-\mathrm{IL} 1 \beta$ axis was shown and the patient was successfully treated by anakinra ${ }^{14}$. Treatment with adalimumab and anakinra are being reported as case presentations $s^{4,14}$, suggesting further evaluation of TNF- $\alpha$ and IL-1 $\beta$ in the pathogenesis. In our case disease modifying anti-rheumatic drug therapies (DMARDs) were sufficient to control disease activity. Biologics were spared to treatment resistant exacerbations.

Because of the rarity of the disease and the unknown etiopathogenetic mechanisms, targeted therapy is still unavailable ${ }^{6}$. Treatment 
options for SAPHO based on case reports or uncontrolled studies including NSAIDs, colchicine, corticosteroids and bisphosphonates, and DMARDs such as methotrexate, sulfasalazine ${ }^{15}$. Bisphosphonates have antiosteoclastic effect and anti-inflammatory action. Antimicrobial therapy are such as azithromycin, doxycycline and sulfamethoxazole/trimethoprim are also used ${ }^{14,15}$. Biologic agents are being used for the patients who are unresponsive to the bisphosphonates, encouraging results were reported with infliximab and etanercept ${ }^{15}$, and recently by adalimumab and anakinra ${ }^{4,14}$. Since the cost effectiveness of biological therapy is not yet clear for SAPHO and no randomized controlled trial is imminent, recommendations for this therapeutic approach remains based on small series of retrospective cases for now ${ }^{4}$. Among all treatment options, NSAIDS are generally considered as first-line treatment in SAPHO ${ }^{12}$. However, combination with the other DMARDs is almost always required ${ }^{15}$. We used methotrexate in combination with NSAID and sulfasalazine as initial treatment for preventing long term steroid exposure. Because of the conflicting data and the lack of the evidence of any infectious agents in the cultures and the biopsy of our patient, we did not use any antibiotics as the first-line therapy in our patient ${ }^{14,15}$.

In conclusion, SAPHO syndrome continues to be a nosologic enigma. It is very rare in the pediatric population. Diagnosis and treatment options are based on the criteria for adults. Subcutaneous methotrexate seems to be a favorable choice in combination with NSAID and sulfasalazine before starting biologic agents. Recognition of SAPHO syndrome is important to avoid prolonged antibiotic treatment of osteo-articular lesions and unnecessary invasive procedures.

\section{REFERENCES}

1. Chamot AM, Benhamou CL, Kahn MF, et al. Acnepustulosis-hyperostosis-osteitis syndrome: Results of a national survey 85 cases. Rev Rheum Mal Osteoartic 1987; 54: 187-196.

2. Hayem G, Bouchard-Chabot A, Benali K, et al. SAPHO syndrome: A long-term follow-up of 120 cases. Semin Arthritis Rheum 1999; 29: 159-171.

3. Aljuhani F, Tournadre A, Tatar Z, et al. The SAPHO syndrome: A single-center study of 41 adult patients. J Rheumatol 2015; 42: 329-334.
4. Eleftheriou D, Gerschman T, Sebire N, et al. Biologic therapy in refractory chronic non-bacterial osteomyelitis of childhood. Rheumatology (Oxford) 2010; 49: 15051512.

5. Beretta-Piccoli BC, Sauvain MJ, Gal I, et al. Synovitis, acne, pustulosis, hyperostosis, osteitis (SAPHO) syndrome in childhood: A report of ten cases and review of the literature. Eur J Pediatr 2000; 159: 594601.

6. Benhamou CL, Chamot AM, Khan MF. Synovitisacne-pustulosis-hyperostosis-osteomyelitis syndrome (SAPHO): A new syndrome among spondyloartropathies? Clin Exp Rheum 1988; 6: 109-112.

7. ColinaM, Govoni M, Orzincolo C, et al. Clinical and radiological evolution of synovitis, acne, pustulosis, hyperostosis, and ostetitis (SAPHO) syndrome: A single center study of a cohort of 71 subjects. Arthritis Rheum 2009; 61: 813-821.

8. Schuster T, Bielek J, Dietz HG, et al. Chronic recurrent multifocal osteomyelitis (CRMO). Eur J Pediatr Surg 1996; 6: 45-51.

9. Jurik AG, Egund N. MRI in chronic recurrent multifocal osteomyelitis. Skeletal Radiol 1997; 26: 230-238.

10. Dilhlmann W, Dihlmann SW, Hering L. Acquired hyperostosis syndrome--AHYS--(sternocostoclavicular hyperostosis, pustuloticarthro-osteitis, SAPHOsyndrome): Bone scintigraphy of the anterior chest wall. Clin Rheumatol 1997; 16: 13-24.

11. Colina M, Lo Monaco A, Khodeir M, et al. Propionibacterium acnes and SAPHO syndrome: a case report and literature review. Clin Exp Rheumatol 2007; 25: 457-460.

12. Matzaroglou C, Velissaris D, Karageorgos A, et al SAPHO syndrome diagnosis and treatment: Report of five cases and review of the literature. The Open Orthop J 2009; 3: 100-106.

13. Hayem G. Valuable lessons from SAPHO syndrome. Joint Bone Spine 2007; 74: 123-126.

14. Colina M, Pizzirani C, Khodeir M, et al. Dysregulation of P2X7 receptor-inflammasome axis in SAPHO syndrome: successful treatment with anakinra. Rheumatology (Oxford) 2010; 49: 1416-1418.

15. Wagner AD, Andresen J, Jendro MC, et al. Sustained response to tumor necrosis factor alpha-blocking agents in two patients with SAPHO syndrome. Arthritis Rheum 2002; 46: 1965-1968. 